\title{
The Wonderful DMARD with Multiple Toxicities
}

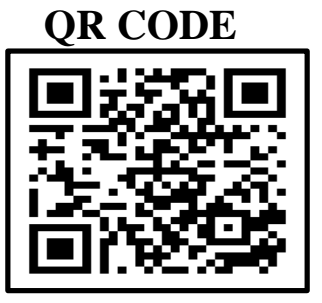

\section{YASHIKA KAUSHAL*1, RATIBHA KAUSHAL ${ }^{2}$, ISHA SHARMA ${ }^{3}$}

Methotrexate is a type of disease-modifying anti-rheumatic drug (DMARD). It is used to reduce activity of the immune system for people who have certain conditions. Methotrexate is a chemotherapy agent and immune system suppressant. Its use may be limited by concerns regarding its adverse reactions. The occurrence of adverse drug reactions in some cases leads to the therapy discontinuation. Although adverse drug reactions (ADR) of methotrexate generally do not pose a serious threat to the health of patients and a reduction in the dose of methotrexate leads to their elimination, in some cases severe toxicities of the drug occur unpredictably. These facts explain the need for close monitoring of the patient's condition and the identification of potential risk factors for drug toxicity on the part of different organs and functional systems. The purpose of this review is to detail about safety and tolerability of methotrexate.

KEYWORDS: Methotrexate, Adverse Drug Reactions, Toxicity, Patient Safety

\section{INTRODUCTION}

Methotrexate is one of the drugs of choice for systemic treatment of moderate to severe forms of multiple disorders. Methotrexate is a highly effective drug that has been used successfully for more than 50 years to treat skin diseases. ${ }^{1}$ At present, methotrexate is also used for the treatment of other dermatological diseases, such as vasculitis, bullous dermatosis, and lymphoproliferative diseases. ${ }^{2}$ Methotrexate has proven to be a highly effective drug, but half of patients experience ADR during treatment. The pathogenesis of most ADR of methotrexate is based on its cytotoxic effect on rapidly dividing body cells, primarily the cells of the bone marrow, gastrointestinal tract, and hepatocytes. Methotrexate toxicity effects all the major organs of the body.

Gastrointestinal disorders are the most common ADR of methotrexate: $18.2 \%$ of patients report nausea and vomiting; $11.1 \%$ have ulcers of the oral mucosa and other mucositis; $7.5 \%$ have abdominal pain; and $6.6 \%$ of patients note intestinal functional disturbances. ${ }^{3}$ The primary mechanism of ADR with methotrexate therapy is inhibition of folate metabolism in tissues with high cell proliferation, with a high need for purines, thymidine, and methionine. Since gastrointestinal epithelium is characterized by a rapid rate of cell population turnover, folate deficiency is the major mechanism in the development of this ADR group. ${ }^{4}$ The incidence of gastrointestinal ADR may be influenced by the patient's genetic characteristics, namely the presence of polymorphisms in the genes encoding the methotrexate transporter proteins.

Hepatotoxicity of methotrexate is one of the most significant ADR of the drug. ${ }^{5}$ The hepatotoxic effect of methotrexate is mainly manifested by two ADR groups: elevated liver enzymes and development of structural changes in the liver. ${ }^{6}$ Liver enzyme elevations occur in $10 \%$ of all patients taking methotrexate on the average.

Liver fibrosis usually develops with a long-term use of methotrexate: such structural changes in the liver occur in $25 \%$ of patients taking methotrexate for more than 5 years. ${ }^{7}$ While testing liver biopsy specimens from patients with rheumatoid arthritis treated with methotrexate, accumulation of polyglutamate forms of methotrexate and folate deficiency was found in cells, which may indicate that the hepatotoxicity of methotrexate is associated with folate depletion due to the action of polyglutamate forms of the drug. ${ }^{8}$ Polyamine synthesis, accumulation of adenosine and deoxyadenosine, impaired metabolism of homocysteine and purines are also thought to play a role in the formation of the hepatotoxic effect of

(C) Yashika Kaushal et al. This is an open access article distributed under the terms of the Creative Commons Attribution License CC-BY-NC 4.o, which permits unrestricted use, distribution and reproduction in any medium, provided the use is not commercial and the original author(s) and source are cited. Submitted on: 26-Sep-2021; Accepted on: 29-Oct-2021 
methotrexate. According to the results of studies in mouse models, the cause of methotrexate-induced liver fibrosis is the stimulation of collagen synthesis by the accumulation of adenosine: adenosine binds adenosine Az receptors on the hepatic fat-storing stellate cells, which are potentially fibrogenic and stimulate collagen production. ${ }^{9}$ Methotrexate triggers the lipid peroxidation process, which leads to the synthesis of reactive oxygen species and damage to liver cells. Possible risk factors for these ADR should be considered when predicting the risk of methotrexate-induced hepatotoxicity.

Other risk factors for increased hepatotoxicity of methotrexate include persistent elevated aminotransferases, alcohol use, chronic liver disease, history of hepatotoxic drug use or exposure to hepatotoxic substances, hereditary predisposition to genetically determined liver diseases, alpha-1 antitrypsin deficiency, and hemochromatosis. ${ }^{6}$ To predict the hepatotoxicity of methotrexate, a complete blood count, liver enzyme testing, serologic studies to identify hepatitis B and C biomarkers, and ruling out other risk factors for increased hepatotoxicity, including genetic ones, are required before starting therapy. Since the drug is excreted by kidneys and impairment of their function may increase the risk of hepatotoxicity, creatinine and urea levels should be assessed. Regular monitoring of aminotransferases as an early and reliable marker of liver injury is now considered sufficient in the first stages of therapy. Additional specific tests to assess liver status are recommended in case of risk factors for hepatotoxicity or abnormal liver chemistry.

Some patients develop hematologic abnormalities, such as myelosuppression manifested by macrocytic anemia, leukopenia, lymphopenia, thrombocytopenia, hypogammaglobulinemia, and pancytopenia. The mechanism of methotrexateinduced thrombocytopenia is based on the release of free oxygen radicals that activate terminal protein kinases, which are involved in the initiation of platelet apoptosis. ${ }^{10}$

The risk factors for hematological toxicity include the elderly, persons with renal insufficiency, hypoalbuminemia, those non-complying with dosing regimen of the drug or folic acid, and alcohol users. Cases of pancytopenia and thrombocytopenia have been described in patients with risk factors with low-dose methotrexate. Patients without risk factors are advised to monitor blood parameters once a week during the first month of methotrexate use and every two weeks during the second and third months. After the fourth month of therapy, in the absence of ADR, the frequency of blood tests can be reduced to every 2-3 months. It should be considered that 4 to 6 weeks after increasing the dose of methotrexate, the risk of hematologic ADR increases, and the patient's state should be closely monitored during this period. Since impaired renal function is one of the most serious predisposing factors for ADR, it is advised to measure the glomerular filtration rate in elderly patients and in those with reduced body weight, even if the blood creatinine and urea nitrogen values are normal. If a patient has impaired renal function, each subsequent methotrexate intake and dose increase should be preceded by test results that do not reveal any negative changes.

Patients taking methotrexate have a higher risk of interstitial pneumonia, pneumocystis pneumonia, and pulmonary fibrosis. ${ }^{6}$ The phenomena of pulmonary toxicity have been observed in patients treated with both low and high doses of the drug, suggesting that the mechanism of toxicity is not conditioned by the methotrexate influence on folate metabolism only." The alleged and investigated mechanisms of the pulmonary toxicity of methotrexate are hypersensitivity reactions, direct cytotoxic effect of methotrexate on lung tissue, activation of mitogen-activated protein kinases, immunosuppression, and impaired cytokine expression, causing an inflammatory response in lung tissues and destruction of lung tissue. ${ }^{12}$ Bronchoalveolar lavage and histological examination of the lung tissue confirm that the pathogenesis of lung injury is based on the hypersensitivity reaction. ${ }^{13}$ Damage to the epithelium and manifestations of fibrosis suggest a direct cytotoxic mechanism of methotrexate effect. ${ }^{14}$

The toxic effect of methotrexate on renal function is a common ADR at high doses of the drug, but in rare cases methotrexate can cause kidney damage leading to kidney failure, even at low doses. Various mechanisms of low-dose methotrexate nephrotoxicity are assumed. Methotrexate has been established to cause renal tubular cell edema and necrosis, leading to irreversible damage to kidney tissue. ${ }^{15}$ The drug is excreted primarily by the kidneys and being relatively insoluble in the acidic medium of urine, may have a direct toxic effect on 
the renal tubular epithelium or precipitate in the renal tubular lumen, causing intratubular obstruction. These processes result in a decreased glomerular filtration rate. Methotrexate causes damage to the cells of the renal glomeruli and renal tubules by increasing the oxidative stress the role of which has been identified in the pathogenesis of chronic kidney disease. The mechanism suggested is an increase in the markers of oxidative stress, 4hydroxynonenal and malonic aldehyde, with longterm use of low doses of methotrexate [16]. Another alleged mechanism of methotrexate-induced nephrotoxicity is decreased blood flow in the renal tissues and consequently impaired renal excretion of water and salts by the kidneys due to the increasing adenosine concentrations in the plasma and intercellular fluid and subsequent activation of adenosine receptors. ${ }^{17}$

Methotrexate affects the body's immune response and increases the risk of opportunistic infections. Cases of pneumocystis pneumonia, atypical actinomycosis, aspergillosis, cryptococcosis, meningitis and herpes virus infections, reactivation of tuberculosis, exacerbations of chronic hepatitis have been described with methotrexate treatment. Weekly low-dose methotrexate has been established to affect $\mathrm{T}$ cell activity ${ }^{18}$, however, it is suggested that an increased risk of infectious diseases may be caused by imbalance in the immune system due to chronic inflammation resulting from the underlying disease or the presence of concomitant disorders and comorbidities.

Methotrexate is prohibited in pregnant women due to the teratogenicity of the drug. Fetal malformations have been observed with methotrexate at all gestational ages, but the most pronounced teratogenic effect of methotrexate is observed during the first 6-8 weeks of pregnancy. ${ }^{19}$ Methotrexate is also prohibited in lactating women because it is found in breast milk and can cause ADR in the baby. Oligospermia, a negative effect of methotrexate on spermatogenesis, has also been described in the literature. ${ }^{20}$

Methotrexate is a risk factor for the development of squamous cell carcinoma of the skin. ${ }^{21}$ It has been found that long-term therapy with methotrexate is associated with the development of Epstein-Barr virus-associated lymphoproliferative diseases, which spontaneously regress after discontinuation of methotrexate. ${ }^{22}$ In addition, a study of the rate of malignancy risk increases has found that patients with rheumatoid arthritis treated with methotrexate were $50 \%$ more likely to have cancer compared to the general population, with five-fold increased risk of non-Hodgkin lymphomas and three-fold increased risk of melanoma and lung cancer.

ADR of mucositis, ulceration of psoriatic lesions, hyperpigmentation, alopecia, toxic epidermal necrolysis, nodulosis, and anaphylaxis have been described in patients taking methotrexate. ${ }^{23}$ One of the skin ADR of the drug is methotrexate-induced nodulosis. It is assumed that the mechanism of formation of these giant cells is based on the methotrexate effect on the increase in adenosine synthesis and its interaction with adenosine $A_{1}$ receptors. ${ }^{24}$ Methotrexate-induced alopecia is thought to be caused by folate deficiency; it rarely occurs with low-dose methotrexate and resolves on its own several months after therapy discontinuation.

At the start of methotrexate therapy, some patients experience headaches, dizziness, weakness and emotional liability. One of the alleged mechanisms of methotrexate-induced neurotoxicity is the effect of increased release of adenosine and its accumulation in the central nervous system. The role of adenosine as a neurotransmitter and neuromodulator in the CNS has been experimentally confirmed: its increased accumulation is associated with the occurrence of headaches, nausea and somnolence. Adenosine has an inhibitory effect in the CNS: when binding to adenosine Ar receptors in the perifornical area of hypothalamus, adenosine can regulate the awakening and falling asleep processes, which may explain the weakness and somnolence that some patients experience after taking methotrexate. ${ }^{25}$ In children treated with high doses of methotrexate, its neurotoxic effect was manifested in the pronounced somnolence and comatose state, which were reversible with theophylline therapy, a nonselective adenosine receptor antagonist. The neurotoxic effect of methotrexate may also be due to excitotoxic death of neurons, which can be caused by homocysteine metabolites: homocysteine acid and cysteine sulfonic acids. ${ }^{26}$ Another possible mechanism may be impaired metabolism of biopterin, leading to a decrease in the synthesis of monoamine neurotransmitters. ${ }^{27}$ 
Rarely, low doses of methotrexate cause osteopathies like bone pain, long bone fractures, and osteoporosis.

Methotrexate-induced osteopathy syndrome was first described as a stress fracture of the lower extremity bones, diffuse bone pain, and osteoporosis in children with acute lymphoblastic leukemia receiving long-term therapy with low-dose methotrexate. Several cases of methotrexateinduced osteopathy in patients with rheumatoid arthritis and psoriasis have been described. An animal study has found that long-term use of lowdose methotrexate causes pronounced osteopenia by reducing osteoblast activity and increasing osteoclast activity. ${ }^{28}$

It should be considered that in some cases, even a single dose of methotrexate may lead to serious and complex ADR. The risk of fatal ADR with methotrexate therapy is small. The toxic effects of methotrexate on the hematopoietic system, respiratory system and liver are most life threatening. Therefore, careful identification of risk factors for increased toxicity of methotrexate and comprehensive monitoring of the patient's condition with regard to these ADR groups is necessary. Hepatologic and hematologic ADR of methotrexate may manifest as weakness, nausea, stomatitis, and syncope in patients. Similar symptoms that develop during methotrexate therapy should be alerted. Careful monitoring of the patient's condition will reduce the risk of ADR. Caution should be exercised in patients with hepatitis, hepatic cirrhosis, and other conditions associated with impaired hepatic function due to its potential hepatotoxicity. Liver parameters should be closely monitored in these patients.

Despite the lack of unambiguous data regarding the complete or partial prohibition on alcohol consumption in the period of methotrexate therapy, history of alcoholism and alcohol-induced liver diseases are relative contraindications to the drug administration. The use of methotrexate may be restricted by administration of other drugs that have toxic effects on the liver. Since the drug is primarily excreted by the kidneys, methotrexate should be used with caution in patients with renal impairment. With impaired renal excretion of methotrexate, plasma concentrations of the drug increase and therefore the risk of its toxicity increases. Close attention should be paid to the state of patients with immunosuppression and chronic infections prone to exacerbation as a result of immunosuppressive effect of methotrexate. In exacerbation of the infectious process, the drug should be temporarily discontinued. Before prescribing methotrexate, it is necessary to assess whether the patient is able to fulfil all prescriptions responsibly, since the therapy success and patient's safety depend largely on careful selection of the dose regimen and duration of therapy, as well as on regular examination for potential ADR.

Often, a potential benefit of therapy may outweigh the possible risks for a patient with a disease or condition that limits the use of methotrexate. Treatment decisions should be made by the physician individually for each patient based on the risk-benefit assessment.

\section{REFERENCES}

1. Edmudson W. Treatment of Psoriasis with Folic Acid Antagonists. Arch Dermatol. 1958;78(2):200.

2. Shen S, O'Brien T, Yap LM, et al. The use of methotrexate in dermatology: a review. Australas J Dermatol. 2012;53(1):1-18.

3. West J, Ogston S, Foerster J. Safety and Efficacy of Methotrexate in Psoriasis: A Meta-Analysis of Published Trials. PLoS One. 2016;11(5):eo153740. https://doi.org/10.1371/journal.pone.0153740

4. Van Ede A, Laan R, Blom H, et al. Methotrexate in rheumatoid arthritis: An update with focus on mechanisms involved in toxicity. Semin Arthritis Rheum. 1998;27(5):277-92.

5. Bedoui Y, Guillot X, Sélambarom J, et al. Methotrexate an Old Drug with New Tricks. Int J Mol Sci. 2019;20(20):5023. https://doi.org/10.3390/ijms20205023

6. Conway R, Carey J. Risk of liver disease in methotrexate treated patients. World J Hepatol. 2017;9(26):1092.

https://doi.org/10.4254/wjh.v9.i26.1092

7. Themido R, Loureiro M, Pecegueiro M, et al. Methotrexate hepatotoxicity in psoriatic patients submitted to long-term therapy. Acta Derm Venereol (Stockh).1992;72:361-4.

8. Kremer J, Galivan J, Streckfuss A, Kamen B. Methotrexate metabolism analysis in blood and liver of rheumatoid arthritis patients: Association with hepatic folate deficiency and formation of polyglutamates. Arthritis Rheum. 1986;29(7):832-5. 9. Chan E, Montesinos M, Fernandez P, et al. Adenosine $\mathrm{A} 2 \mathrm{~A}$ receptors play a role in the 
pathogenesis of hepatic cirrhosis. Br J Pharmacol. 2006;148(8):1144-55.

https://doi.org/10.1038/sj.bjp.0706812

10. Paul M, Hemshekhar M, Thushara R, et al. Methotrexate Promotes Platelet Apoptosis via JNKMediated Mitochondrial Damage: Alleviation by NAcetylcysteine and N-Acetylcysteine Amide. PLoS One. 2015;10(6):e0127558.

https://doi.org/10.1371/journal.pone.0127558

11. Olsen E. The pharmacology of methotrexate. J Am Acad Dermatol. 1991;25(2):306-18.

12. Kim Y, Song M, Ryu J. Inflammation in methotrexate-induced pulmonary toxicity occurs via the p38 MAPK pathway. Toxicology. 2009;256(3):183-90.

https://doi.org/10.1016/j.tox.2008.11.016

13. Lateef $\mathrm{O}$, Shakoor N, Balk R. Methotrexate pulmonary toxicity. Expert Opin Drug Saf. 2005;4(4):723-30.

https://doi.org/10.1517/14740338.4.4.723

14. Ohbayashi M, Suzuki M, Yashiro Y, et al. Induction of pulmonary fibrosis by methotrexate treatment in mice lung in vivo and in vitro. J Toxicol Sci.

https://doi.org/10.2131/jts.35.653

2010;35(5):653-61.

15. Grönroos M, Chen M, Jahnukainen T, Capitanio A, Aizman R, Celsi G. Methotrexate induces cell swelling and necrosis in renal tubular cells. Pediatr Blood Cancer. 2006;46(5):624-9. https://doi.org/10.1002/pbc.20471

16. $\mathrm{Li} \mathrm{X}$, Abe $\mathrm{E}$, Yamakawa Y. Effect of Administration Duration of Low Dose Methotrexate on Development of Acute Kidney Injury in Rats. J Kidney. 2016;2(3):130. https://doi.org/10.4172/24721220.1000130

17. Cronstein B. The mechanism of action of methotrexate. Rheum Dis Clin North Am. 1997;23(4):739-55.

18. Genestier L, Paillot R, Fournel S, Ferraro C, et al. Immunosuppressive properties of methotrexate: apoptosis and clonal deletion of activated peripheral T cells. J Clin Invest. 1998;102(2):322-8.

19. Lloyd M. The effects of methotrexate on pregnancy, fertility and lactation. QJM. 1999;92(10):551-63. 2o. Buckley L, Bullaboy C, Leichtman L, Marquez M. Multiple congenital anomalies associated with weekly low-dose methotrexate treatment of the mother. Arthritis Rheum. 1997;40(5):971-3.

21. Stern R, Laird N. The carcinogenic risk of treatments for severe psoriasis. Cancer. 1994;73(11):2759-64.

22. Kamel O, van de Rijn M, LeBrun D, et al. Lymphoid neoplasms in patients with rheumatoid arthritis and dermatomyositis: Frequency of Epstein-Barr virus and other features associated with immunosuppression. Hum Pathol. 1994;25(7):63843.

23. Kalantzis A, Marshman Z, Falconer D, et al. Oral effects of low-dose methotrexate treatment. Oral Surg Oral Med Oral Pathol Oral Radiol Endod. 2005;100(1):52-62.

https://doi.org/10.1016/j.tripleo.2004.08.020.

24. Merrill J, Shen C, Schreibman D, et al. Adenosine A1 receptor promotion of multinucleated giant cell formation by human monocytes. A mechanism for methotrexate-induced nodulosis in rheumatoid arthritis. Arthritis Rheum. 1997;40(7):1308-15.

25. Thakkar M, Engemann S, Walsh K, Sahota P. Adenosine and the homeostatic control of sleep: Effects of Ar receptor blockade in the perifornical lateral hypothalamus on sleep-wakefulness. Neuroscience. 2008;153(4):875-80.

26. Quinn C, Griener J, Bottiglieri T, Hyland K, Farrow A, Kamen B. Elevation of homocysteine and excitatory amino acid neurotransmitters in the CSF of children who receive methotrexate for the treatment of cancer. J Clin Oncol. 1997;15(8):280o-6. 27. Millot F, Dhondt J, Mazingue F, Mechinaud F, Ingrand $\mathrm{P}$, Guilhot $\mathrm{F}$. Changes of Cerebral Biopterin and Biogenic Amine Metabolism in Leukemic Children Receiving $5 \mathrm{~g} / \mathrm{m} 2$ Intravenous Methotrexate. Pediatr Res. 1995;37(2):151-4.

28. May K, West S, Mcdermott M, Huffer W. The Effect of Low-Dose Methotrexate on Bone Metabolism and Histomorphometry in Rats. Arthritis Rheum. 1994;37(2):201-6. 
Cite this article as:

Kaushal Y, Kaushal R, Sharma I. The Wonderful DMARD with Multiple Toxicities. Int

Source of support: Nil, Conflict of interest: None declared

Healthc Res J. 2021;5(7):RV5-RV10. https://doi.org/10.26440/IHRJ/0507.10470

AUTHOR AFFILIATIONS: $\left({ }^{*}\right.$ Corresponding Author)

1. MBBS, Presently Clinical Observer, International Medical Graduate, Unit Number 322, Building Number 8068 , 120 A Street, Surrey, British

Columbia, Canada, Postal Code- $\mathrm{V}_{3} \mathrm{~W}_{3} \mathrm{P}_{3}$

2. International Medical Graduate, British Columbia, Canada

3. Private Practitioner, Patiala

Contact Corresponding Author At: yashika394kaushal[at]gmail[dot]com 